\title{
Il trattamento dei detenuti sex offenders nel carcere di Castelvetrano (TP)
}

\author{
Angela Adragna
}

\begin{abstract}
Riassunto
L'articolo è incentrato sull'analisi del programma trattamentale offerto ai detenuti sex offenders nella Casa Circondariale di Castelvetrano (Trapani), nel periodo 2009-2012, attraverso il progetto "www...parliamonepure".

L'analisi ha consentito in particolare di evidenziare come i detenuti sex offenders, nonostante la loro "ricercata invisibilità" nel contesto penitenziario, abbisognino di interventi specifici, intramurari ed extramurari, volti ad evitare una futura recidiva.

In tal senso si auspica che le attività trattamentali di tipo psico-sociale di gruppo nel contesto restrittivo possano essere svolte con logica di continuità, anche se in spazi e tempi diversi, affinché il detenuto possa essere adeguatamente supportato sia all'interno del carcere che all'esterno, con presa in carico da parte degli Uffici di Esecuzione Penale Esterna (U.E.P.E.) nelle prime fasi del reingresso in ambiente libero tramite interventi di assistenza post-penitenziaria e di invio eventuale alle strutture sanitarie di riferimento.
\end{abstract}

\section{Résumé}

L'article est consacré à l'analyse du programme de traitement offert aux délinquants sexuels dans la prison de Castelvetrano, de 2009 à 2012, dans le cadre du projet « Venez nous en parler » ("www...parliamonepure”).

L'analyse a montré que les délinquants sexuels, malgré leur «requête d'invisibilité » dans la prison, ont besoin de mesures spécifiques, en milieu carcéral et à l'extérieur, pour prévenir la récidive.

Dans ce contexte, il faut espérer que les mesures de resocialisation psychosociales puissent être réalisées avec continuité en prison et hors de prison. À l'extérieur de la prison, le condamné sera pris en charge par le service de probation (UEPE - Ufficio per l'Esecuzione Penale Esterna) au cours des premières phases de sa réinsertion et des soins médicaux lui seront offert, si besoin est.

\section{Abstract}

The article focuses on the analysis of a treatment program provided to sex offenders in Castelvetrano Prison, from 2009 to 2012, as part of the project "Come and talk to us" ("www...parliamonepure").

In particular, the analysis showed that sex offenders, despite their "requested invisibility" in the prison, need specific actions, inside and outside prison walls, in order to avoid the recidivism.

In this respect, it is to be hoped that psycho-social resocialisation programs can be carried out ensuring continuity both inside and outside the prison walls. Outside the prison walls, the offender would be taken over by probation services during the first phases of their re-entry to the society through post-penitentiary assistance and subsequently health care treatment if needed.

Key words: sex offenders; prison inmates; recidivism; probation services; resocialization programs.

\section{Introduzione.}

Il presente articolo perviene, attraverso un percorso di riflessione teorica, di ricognizione legislativa, di analisi conoscitiva e di sintesi propositiva, alla validazione di uno strumento utilizzato nell'intervento con i detenuti così detti sex offenders, il progetto www.parliamonepure..., sperimentato dalla scrivente presso la Casa Circondariale di Castelvetrano (Trapani).

Questa tipologia di detenuti negli ultimi anni è stata oggetto dell'attenzione di studiosi e di operatori sia per la necessità di individuare risposte finalizzate al contenimento del fenomeno, sia per intervenire sul piano della riabilitazione e del recupero sociale.

\footnotetext{
• Funzionario della Professionalità di Servizio Sociale presso l'Ufficio di Esecuzione Penale Esterna di Trapani.
} 
E' su questa linea di indirizzo che le direttive europee e successivamente la legge n. 38/2009 "Recante misure urgenti in materia di sicurezza pubblica e di contrasto alla violenza sessuale, nonché in tema di atti persecutori" hanno posto l'attenzione dal punto di vista legislativo.

Una prima azione progettuale promossa dal Ministero della Giustizia (2001) Amministrazione Penitenziaria - riguarda il progetto W.O.L.F., e successivamente il progetto "For W.O.L.F.", che ha fatto da apriprista per l'avvio di una politica orientata alla formazione degli operatori penitenziari al fine di attuare percorsi riabilitativi per i detenuti sex offenders. Sulla base di tali progetti sono state successivamente avviate alcune esperienze rivolte al trattamento degli autori di reati sessuali presso alcuni istituti penitenziari italiani, come la Casa di Reclusione di Milano "Bollate", le Case Circondariali di Bari, di Bologna, di Palermo, di Prato, di Torino, di Verbania, di Vercelli e non ultima quella di Castelvetrano.

Nel 2009 il gruppo degli operatori dell'istituto penitenziario di Castelvetrano ha elaborato e realizzato un progetto d'intervento che ha costituito l'occasione per una seria riflessione sulla tematica, sulle teorie di riferimento e sulle modalità operative da attuare nei confronti degli autori di reati sessuali, attraverso un'analisi del contesto sotto il profilo ordinamentale e teorico e uno studio della realtà locale dell'istituto penitenziario, delle risorse trattamentali disponibili e di quelle professionali e umane, nonché dei destinatari dell'intervento.

\section{Breve analisi del contesto sociale di Castelvetrano.}

Assume sempre più forza il convincimento che l'esecuzione della pena sia strettamente legata al territorio in cui nascono e si sviluppano i fenomeni di devianza. Dunque, si può affermare che il carcere di Castelvetrano appartiene alla sua città e al relativo sistema dei servizi. Detta struttura penitenziaria è ubicata nella periferia della città, a 90 chilometri da Trapani, capoluogo della provincia.

Il territorio di Castelvetrano presenta una forte concentrazione di servizi socio-sanitari rispetto agli altri comuni, come Salaparuta, Poggioreale, Santa Ninfa, Partanna e Campobello di Mazara, tutti facenti parte del Distretto socio-sanitario n. 54, di cui Castelvetrano è Comune capofila.

Per quanto riguarda l'assetto demografico, questo ha subito una profonda trasformazione dopo il sisma del 1968, in particolare nei Comuni di Salaparuta, Poggioreale e Santa Ninfa. Il fenomeno dell'emigrazione ha assunto un carattere di vero e proprio esodo che ha interessato per lo più giovani e nuclei familiari. Di contro, si è registrata un'elevata percentuale di popolazione anziana, soprattutto nei comuni di minori dimensioni. L'allungamento della vita media ed il movimento migratorio dei giovani hanno creato uno squilibrio nella struttura demografica, con la contrazione delle fasce giovanili e l'aumento di quelle adulte. Si è, pertanto, moltiplicato il numero di anziani che vivono da soli e che soprattutto dispongono di esigue reti parentali. Relativamente all'anno 2014 la popolazione residente è pari a 31.719 abitanti ${ }^{1}$.

\footnotetext{
${ }^{1}$ www.demo.istat.it
} 
L'assetto socio-economico risulta condizionato dall'alto tasso di disoccupazione, legato probabilmente al rallentamento della ricostruzione edilizia e allo scarso sviluppo dell'economia artigianale che risente della grande produzione. Nell'ambito del tessuto economico locale, l'agricoltura continua a svolgere un ruolo preminente, mentre lo sviluppo industriale se, da un lato, ha favorito l'economia familiare, dall'altro ha ridotto le possibilità di impiego ed in particolare di utilizzo della manodopera. Altro bacino potenziale di crescita economica è rappresentato dal turismo e dalla valorizzazione del patrimonio culturale, monumentale ed archeologico, sebbene allo stato attuale risultino insufficienti le iniziative per lo sviluppo di un vero polo d'interesse turistico e lavorativo.

Rispetto al contesto sociale, si rileva una situazione di "doppia povertà" che si manifesta come economica, per la precarietà dell'attività lavorativa, nonché culturale, per l'alto numero di analfabeti e/o senza titolo di studio e per la marginalità legata all'assenza e alla distanza dai centri di distribuzione delle risorse culturali, economiche, sociali e politiche.

Per le ragioni sopra esposte, bassa è la natalità tra la fascia sociale produttiva, quest'ultima sempre più ridotta.

Il fenomeno dell'immigrazione, prevalentemente di provenienza nord africana, caratterizzato dalla trasversalità e da aspetti di dequalificazione nel mercato del lavoro, assume un carattere complementare piuttosto che sostitutivo/competitivo, incanalandosi in segmenti meno coperti dall'offerta locale di lavoro. Dunque, tale fenomeno evoca, assai debolmente, il pericolo di una competizione che sottrae lavoro e reddito ai cittadini locali.
Tuttavia, il carattere dequalificato della loro offerta lavorativa espone questi immigrati al rischio di una maggiore contiguità con il lavoro sommerso e, talvolta, all'inserimento in canali di lavoro illegale legati al crimine individuale ed organizzato.

In merito al fenomeno della criminalità sanzionata, sulla base dei dati rilevati e gestiti dall'U.E.P.E. (Ufficio per l'Esecuzione Penale Esterna) di Trapani, si registrano per l'anno 2014, nel distretto di Castelvetrano, complessivamente 182 casi.

$\mathrm{Va}$ rilevato che l'età media dei soggetti condannati è compresa nella fascia tra i 30 ed i 40 anni; la percentuale di genere propende in maniera consistente per il sesso maschile; per tale fascia di soggetti la scolarizzazione è assai modesta, attestandosi prevalentemente sulla licenza di scuola elementare; lo strato sociale di provenienza è prevalentemente quella delle sacche di marginalità legate alla particolare ubicazione del territorio periferico, agli scarsi collegamenti con la provincia ed alla prevalente estrazione rurale della popolazione.

Per quanto attiene alle caratteristiche dei reati commessi, si evidenziano i reati di criminalità organizzata, caratterizzati dal radicamento nel territorio del fenomeno mafioso, i reati contro il patrimonio, causati dalla già detta condizione di marginalità ed i reati strettamente correlati alla problematica della tossicodipendenza e a tutto ciò che gravita attorno ad essa² .

\section{L'istituto penitenziario di Castelvetrano.}

Al 31 dicembre 2015, la struttura penitenziaria ospita 69 detenuti che, nella maggior parte dei

\footnotetext{
${ }^{2}$ www.ComunediCastelvetrano.it
} 
casi, non sono residenti sul territorio di Castelvetrano bensì provenienti da altre realtà territoriali.

Negli anni di sperimentazione del progetto "www...parliamone pure" (2009-2012), il carcere ospitava in media 100 detenuti e la sezione protetti, aperta nell'ottobre del 2008, accoglieva 33 sex offenders di cui circa 20 aderenti all'iniziativa progettuale.

L'Istituto Penitenziario è così strutturato:

- Sezione Nuovi Giunti, per persone che, per la prima volta, fanno ingresso nell'istituto penitenziario sia che provengano dalla libertà, sia che provengano da un altro istituto per trasferimento temporaneo o assegnazione definitiva.

- Sezione Comuni, per detenuti che sono stati condannati nel massimo a cinque anni di reclusione.

- Sezione Alta Sicurezza, per detenuti condannati per reati associativi di tipo mafioso, per terrorismo, per violazione legge stupefacenti (oltre cinque anni di reclusione).

- Sezione Protetti, per detenuti che si sono resi responsabili di reati di natura sessuale, ma anche per coloro che chiedono una particolare tutela a causa della propria posizione giuridica o personale. Essi generalmente sono esclusi dalle attività ricreative, culturali, lavorative e di formazione professionale in comune con altri detenuti per garantire la loro sicurezza. Detti detenuti accedono soltanto alle limitate occasioni promosse esclusivamente per loro.

- Sezione Semiliberi, per condannati e internati, che abbiano scontato almeno metà della pena (o i $2 / 3$ per i reati più gravi) e che trascorrono una parte della giornata fuori dall'istituto di pena per partecipare ad attività lavorative, istruttive o comunque utili al reinserimento sociale.

- La Sezione Donne non è presente.

L'équipe della struttura penitenziaria è composta dal Direttore, dal Comandante della Polizia Penitenziaria, da due Funzionari della Professionalità Giuridico-Pedagogica, da un Sanitario, da due Funzionari della Professionalità di Servizio Sociale, appartenenti all'U.E.PE. di Trapani, che forniscono consulenza per l'attività di osservazione e supporto al trattamento penitenziario, da un esperto psicologo (ex art. 80 O.P.), dal Cappellano ed eventualmente da altri operatori che collaborino al trattamento.

Il lavoro di équipe si conclude con la stesura di una relazione di sintesi, che contiene il programma di trattamento individualizzato. Questo documento può anche essere predisposto per fornire dati utili alla Magistratura di Sorveglianza in occasione di richiesta di misura alternativa o di altri benefici formulata dalla persona detenuta.

\section{Descrizione del progetto "www...parliamone...ppure".}

Il progetto "wmw...parliamone....pure", attivato all'interno dell'istituto penitenziario di Castelvetrano, nasce nell'anno 2009 ed ha visto un prosieguo in cinque diverse fasi. Detto progetto è stato rivolto esclusivamente alla popolazione dei detenuti della "Sezione Protetti" che, sin dalla sua apertura, accoglie prevalentemente condannati per reati sessuali.

L'intendimento della Direzione della Casa Circondariale è stato quello di coinvolgere gli 
operatori nella individuazione e nella progettazione di azioni e di attività trattamentali specifiche per i sex offenders.

Sin dall'inizio, il progetto è stata curato dall'Ufficio Educatori dell'istituto, dagli assistenti sociali dell'Ufficio di Esecuzione Penale Esterna di Trapani e dall'esperto psicologo (ex art. 80 O.P.) operante all'interno della Casa Circondariale.

Lo sguardo degli operatori, in una fase propedeutica, si è focalizzato su tre aspetti:

- la particolare natura dei reati sessuali, assolutamente distanti dai reati così detti "comuni";

- il ruolo assunto dal carcere nei confronti di questa tipologia di condannati;

- $\quad$ il potenziale ruolo trattamentale svolto dagli operatori del carcere e finalizzato all'avvio di un processo di revisione critica e, ove possibile, di preparazione all'invio verso servizi socio-sanitari territoriali.

Il ruolo degli operatori penitenziari può rappresentare un punto di "aggancio" degli autori di reati sessuali per l'avvio di un percorso di riflessione, di approfondimento, di contatto che gli stessi autori di reato possono realizzare sul piano emozionale ed empatico ed inoltre l'inizio di un percorso di richiesta di aiuto da proseguire anche fuori dal carcere a conclusione della pena detentiva.

Il progetto, come già anticipato, è stato rivolto ai detenuti della sezione protetti, condannati per delitti sessuali e differenziati per reati commessi a danno di minori e per reati commessi a danno di donne.

Il paradigma teorico adottato è quello sistemicorelazionale sulla base del quale, oltre la specifica condotta criminale agita, va osservata la totalità della persona.

L'obiettivo generale del progetto è stato quello di garantire ai sex offenders opportunità trattamentali miranti all'evoluzione sia del singolo che del "gruppo contesto", in un'ottica di riabilitazione, risocializzazione, riconoscimento e assunzione di responsabilità rispetto alla vittima e al danno arrecatole.

Gli obiettivi specifici sono stati individuati e definiti nel corso delle cinque fasi progettuali in relazione allo sviluppo delle capacità espressive e di consapevolezza di sé dell'intero gruppo e dei singoli. In particolare, sono stati apprezzati notevoli evoluzioni in merito alle risonanze emotive e all'empatia nel gruppo.

Si è attuata una metodologia di intervento integrato psico-socio-educativo attraverso lo strumento del lavoro di gruppo e delle tecniche dell'ascolto, confronto, dibattito, brain storming.

Le azioni del progetto si sono tradotte solo in incontri di gruppo tenuti all'interno della sezione con la partecipazione dei detenuti che hanno aderito a seguito di colloquio individuale o di avviso dato alla popolazione detenuta nella sezione protetti. I gruppi sono stati divisi per tipologia di reato distinguendo il "gruppo pedofili" da quello di coloro che avevano stuprato delle donne; il numero è variato da un minimo di 12 ad un massimo di 18 detenuti per singolo gruppo; la cadenza degli incontri è stata stabilita di volta in volta in relazione alle cinque fasi del progetto in accordo con la Direzione della Casa Circondariale e con l'Ufficio Comando; le attività hanno avuto una durata di circa un'ora e mezza per singolo gruppo. 


\subsection{Fase operativa.}

Il progetto è stato portato avanti in cinque diverse fasi e, per ognuna di esse, l'équipe degli operatori implicati ha prodotto reports indicanti le singole azioni compiute, gli strumenti e le tecniche operative impiegate, gli obiettivi perseguiti, le criticità riscontrate, le valutazioni tecnico-professionali circa i risultati conseguiti e gli obiettivi per le successive fasi.

La prima fase del progetto ha previsto:

- la selezione dei sex offenders detenuti nella III "Sezione protetti" che, all'epoca, ospitava 33 persone;

- la distinzione fra i condannati in base alle vittime dei reati commessi, differenziando cioè $\mathrm{i}$ pedofili da coloro che avevano violentato delle donne;

- la realizzazione di colloqui conoscitivi volti ad informare il condannato circa la nuova attività ed a individuare la disponibilità dello stesso a partecipare al progetto;

- l'individuazione di una metodologia orientata all'ascolto, alla conoscenza di se stessi, al riconoscimento delle proprie emozioni;

- l'individuazione di strumenti operativi;

- la selezione dei detenuti che, sulla base di caratteristiche personologiche, del livello di maturità psicologica e del grado di consapevolezza, sono stati ritenuti idonei per un proficuo svolgimento delle attività gruppali.

Gli incontri di gruppo sono stati così articolati:

${ }^{3}$ Marmocchi P., Dall'Aglio C., Tannini M., Educare le life skills, Edizione Centro Studi Erickson, Trento, 2004.
- Presentazione degli operatori del progetto e successiva presentazione di ogni singolo componente;

- Riflessione e trattazione del concetto del sé, legato al reato commesso, ma non solo, e sul singolo percorso giudiziario; somministrazione ad ogni detenuto della scheda dal titolo "Parla di Te"4;

- Trattazione del "concetto di aiuto" e delle correlate emozioni; somministrazione della scheda dal titolo "Riconoscere le emozioni degli altri”;

- Prosecuzione della riflessione sul tema dell'aiuto e sul concetto dei comportamenti che mettono a rischio un soggetto; somministrazione della scheda dal titolo "Rischio";

- Riflessione sulla percezione dell'altro nei propri confronti, sull'autopercezione ed attenzione alle emozioni scaturite; somministrazione della scheda dal titolo "Un amico mi descrive";

- Argomentazione sulla capacità relazionale, riflessione sullitem riferito alla gestione del conflitto e somministrazione della relativa scheda;

- Attività di cineforum con successivo dibattito.

Nella prima fase, l'obiettivo centrale è stato quello di accompagnare i sex offenders verso un percorso inizialmente non centrato sul reato, ma piuttosto sul riconoscimento delle proprie competenze e capacità di vita, favorendo la sperimentazione del sé sociale.

\footnotetext{
${ }^{4}$ Ibidem.
} 
L'attività di gruppo è stata, poi, orientata verso il reato commesso ed è stata riferita non solo alle conseguenze provocate nella vita del detenuto dalla vicenda giudiziaria, ma anche alle ripercussioni sulla vittima del reato e sulla società civile.

Nella seconda fase, gli incontri di gruppo sono stati così articolati:

- Attività di cineforum con successivo dibattito;

- Riflessione sulla percezione propria e del mondo esterno rispetto alla tipologia di reato;

- Approfondimento rispetto allo specifico reato di violenza sessuale con particolare riguardo alla recidiva; attenzione rivolta verso la progettualità del sex offender nella comunità civile;

- Valutazione in itinere del percorso progettuale.

La metodologia che ha caratterizzato l'evoluzione dei lavori di gruppo è stata orientata a stimolare $\mathrm{i}$ detenuti ad una partecipazione attiva che li ha visti interagire con spirito di riflessione, criticità ed approfondimento circa i seguenti contenuti: la concezione e la visione dell'abuso come cicatrice non cancellabile; l'impatto emotivo verso il reato di pedofilia; l'attenzione all'autore del reato di abuso considerata come persona malata e per questo poco consapevole della colpevolezza; l'importanza della "cura" come prevenzione; l'attenzione all'abusante consapevole della propria colpevolezza; l'abuso come cicatrice da poter "riparare"; la richiesta di aiuto nel caso in cui la persona percepisca la difficoltà a gestire i suoi istinti; il ruolo del contesto socio-familiare nella vicenda-reato; l'eventuale esperienza di abuso o molestia subita come potenziale spinta verso una sessualità deviata; il concetto di recidiva nei casi di reati sessuali; percezione propria ed esterna del reato sessuale; autogiudizio e giudizio della comunità civile verso il condannato per reati sessuali; preoccupazione e paura per il re-ingresso nella società civile; stigmatizzazione sociale del condannato per violenza sessuale; richiesta di alcuni detenuti circa l'approfondimento peritale in sede processuale; il concetto di rischio; il confine tra comportamento a rischio e possibilità di un'azione deviante.

Nella terza fase, sono stati costituiti due gruppi, uno composto dai precedenti detenuti partecipanti ed un secondo gruppo composto dai nuovi ingressi.

Gli incontri di gruppo sono stati strutturati non più sulla tipologia di reato commesso (pedofilia e violenza sessuale sulle donne), ma sulla partecipazione gruppale all'esperienza precedente. Tale scelta è scaturita dalla considerazione che andava garantita ai "vecchi" partecipanti la prosecuzione dell'esperienza con un livello di maturazione sempre più consapevole ed orientato ad un auspicato approfondimento circa la tipologia di reato commesso. Diverso è apparso lo status dei nuovi ingressi ancora da motivare all'esperienza gruppale. In questa nuova fase l'équipe ha individuato obiettivi ed attività che, in linea con quanto previsto nell'originario progetto, potessero garantire una continuità al lavoro di riflessione e di approfondimento.

Gli obiettivi di questa terza fase sono stati: avviare una più approfondita conoscenza di se stessi, sostenendo la scoperta e l'esposizione 
degli stati d'animo; stimolare una presa di coscienza rispetto alla reattività individuale nelle diverse situazioni di vita; stimolare la riflessione sul valore della persona e sul rispetto del genere umano; sollecitare verso l'espressione di critiche su di sé e sugli altri; stimolare l'autopercezione verso se stessi e gli altri; spingere la riflessione sui comportamenti a rischio; lavorare sulla stabilità emotiva; favorire la riflessione sull'essere portatore di un bisogno; promuovere la solidarietà di gruppo, l'empatia, la capacità di "chiedere aiuto" a conclusione dell'espiazione della pena ai servizi competenti; favorire una dinamica di gruppo volta alla condivisione ed al sostegno reciproco.

Le attività effettuate riguardano incontri di gruppo in presenza dell'équipe, attività di cineforum e successivo dibattito, verifica in itinere sull'andamento del percorso progettuale.

La metodologia attuata, così come nei precedenti moduli esperienziali, non è stata centrata sulla costituzione di un lavoro predefinito, ma orientata a stimolare e a mantenere fra $i$ partecipanti un confronto sempre più aperto in termini di dinamica emotiva interiore. Ciò al fine di creare un clima di fiducia, di solidarietà e di condivisione tra i partecipanti ed i componenti l'équipe, permettendo così l'avvio di una apertura al dialogo sulla tematica-reato. Dall'osservazione condotta si è evidenziata la reale partecipazione dei detenuti alla messa in discussione sia dei propri comportamenti che del disvalore del commesso reato di tipo sessuale. Tuttavia, tale percorso di rivisitazione, in questa fase, appariva ancora immaturo tanto che l'atteggiamento spesso portato avanti dai soggetti era ancorato su una personale posizione vittimistica nei confronti del sistema di giustizia dal quale si sentivano penalizzati ed il cui esito è stato quello di aver interrotto il loro progetto di vita, senza alcuna riflessione rispetto all'interruzione del percorso di vita della vittima delle proprie azioni.

Sono state poi affrontate tematiche relative alla giustizia sociale, al sistema valoriale, alla cultura deviante ed ai comportamenti a rischio. Anche l'attività di cineforum si è concentrata su problematiche di carattere sociale che, partendo dall'emarginazione della donna e dalla sottomissione di questa all'uomo, potesse far riflettere sul senso di responsabilità e di rispetto nei confronti degli esseri umani tutti.

Nella quarta fase, è stata mantenuta la medesima e precedente composizione dei gruppi dei detenuti, ad eccezione di nuovi inserimenti avvenuti nell'arco dell'esperienza.

Ancora una volta, l'équipe ha individuato obiettivi ed attività, garantendo continuità all'esperienza precedente e spostando, tuttavia, l'ottica dalla riflessione generale sulla persona e sui suoi bisogni emotivi (abilità di vita, di stati emozionali inerenti la sfera quotidiana), elementi centrali delle fasi precedenti, per giungere all'analisi delle storie di vita dei soggetti partecipanti ed ai diversi vissuti come individui e come autori di reato sessuale.

Gli obiettivi di questa quarta fase sono stati: favorire la persistenza di un clima di gruppo accogliente e rispettoso fra i componenti; prendere in esame le storie di vita personale di ogni singolo detenuto; analizzare gli avvenimenti più significativi nel passato del detenuto; "entrare dentro" i comportamenti a rischio agiti dai partecipanti nella loro esperienza di vita; "entrare in contatto" con la stabilità/instabilità emotiva personale; favorire la riflessione 
sull'essere portatore di un bisogno; sollecitare la capacità di "chiedere aiuto" a conclusione dell'espiazione della pena ai servizi competenti.

La quarta fase si è articolata nelle seguenti attività: incontri di gruppo in presenza dell'équipe; incontri con il personale della Polizia Penitenziaria. L'équipe ha individuato alcune parole chiave come: affetto, sicurezza, infanzia, autorità e ogni detenuto ha utilizzato in maniera partecipativa la tecnica proposta del brain storming. Senza reticenze, ha raccontato di sé, approfondendo aspetti, talora intimi, della sua storia di vita familiare, lavorativa e sociale. Ognuno ha esplicitato, anche con sofferenza, aspetti significativi emozionali che hanno caratterizzato la propria evoluzione di vita, a partire dalla prima infanzia, fino alla maturazione ed alla commissione del reato sessuale.

In tale lavoro anche gli operatori hanno scelto di partecipare attivamente al brain storming, agevolando il clima di condivisione e di valorizzazione del detenuto come persona e non come reo sia pur limitatamente all'attività descritta.

Negli ultimi incontri, l'équipe ha proceduto con la restituzione del lavoro svolto al gruppo, evidenziando i seguenti aspetti significativi emersi: la funzione del gruppo come specchio; il coinvolgimento partecipativo di tutti i componenti; una comunicazione reciproca che è apparsa "senza maschera"; la capacità di esplicitare e condividere importanti parti di sé; il rincodurre a sé la capacità di discernimento tra lecito ed illecito; la capacità di dialogare su tematiche legate alla sfera della sessualità.

Anche al gruppo dei detenuti è stata chiesta la restituzione rispetto all'esperienza. Tutti hanno concordato sulla validità del lavoro trattamentale, affermando di "essersi sentiti accolti come persone" e di "aver avuto voce" in una realtà penitenziaria particolare quale la sezione protetti. Alcuni di essi si sono attivati nell'espressione dei propri sentimenti scrivendo, e condividendo con il gruppo, lettere e poesie.

Nella quinta fase, la popolazione detenuta presa in esame è stata pari a 33 detenuti, suddivisi in base alla tipologia del reato, nello specifico 18 detenuti per il primo gruppo (condannati per pedofilia) e 14 detenuti per il secondo gruppo (condannati per violenza sessuale su donne).

L'équipe è stata costituita, come nelle precedenti esperienze, dai medesimi operatori: psicologo, assistenti sociali, educatori, ed ha visto anche la presenza dello psichiatra dell'Azienda Sanitaria Provinciale di Trapani n.9.

Nella definizione degli obiettivi e della relativa programmazione delle attività, è stato necessario tenere conto della presenza numerosa di detenuti che, di recente ingresso, non avevano partecipato alle precedenti fasi del progetto. Per tale ragione, allo scopo di garantire la continuità tra le diverse esperienze, si è pensato di disporre del contributo dei "vecchi" partecipanti, divisi in entrambi i gruppi, come figure trainanti in grado di condurre all'apertura e all'integrazione tra $\mathrm{i}$ “nuovi” ed i "vecchi" partecipanti all'interno di un gruppo che assume così una nuova identità. Ciò, ad avviso degli operatori, tenuto conto degli esiti delle fasi progettuali precedenti, ha reso possibile il consolidamento nel tempo di una matrice di gruppo fondata sulla fiducia reciproca e sulla capacità di ascolto non pregiudiziale, elementi questi di assoluta validità tenuto conto della particolarità della tematica affrontata e degli esiti delle fasi progettuali precedenti.

Gli incontri sono stati così articolati: 
- Conduzione del gruppo verso processi di autoriflessione e direzionamento sulla specificità del tema, avviando la riflessione sui temi del perdono e della vendetta, favorendo il confronto e lo scambio delle esperienze e dei vissuti individuali; ciò con l'obiettivo di promuovere atteggiamenti di reciprocità in seno al gruppo;

- Sollecitazione fornita dal gruppo rispetto al tema della "maschera sociale": maschera della vergogna, della pietà, del nascondersi, della dignità, del coraggio, dell'ipocrisia, del falso sé, dell'ostentazione del coraggio, riconosciuta la sua funzione difensiva nel regime di vita comune; si è avviata, altresì, la riflessione sul tema della violenza come condotta aggressiva eterodiretta con particolare attenzione al tema del danno inflitto;

- Si è affrontato il tema del codice di norme implicite comune alla popolazione detenuta ed alla responsabilità personale nelle forma di adesione a tale codice;

- Drammatizzazione sui seguenti temi: comunicazione disfunzionale ovvero rifiuto e disconferma, al fine di far emergere l'attenzione specifica sull'identità della vittima e sul danno da questa subìto per responsabilità propria; possibilità di "essere perdonati" e, quindi, riflessione sul tema della "riconciliazione" che spesso risponde all'esigenza individuale di recuperare "parti buoni di sé" e riparare al danno stesso, laddove possibile, arrecato alla vittima, anche se difficilmente la riconciliazione potrà essere loro concessa dalla vittima stessa;

- Attività di cineforum per entrambi i gruppi sul tema della prevaricazione agita in solitudine o in gruppo. Si è lavorato sulla capacità personale di autodeterminarsi: cosa si è in grado di fare quando si ha di fronte qualcuno che gestisce un potere abusandone e mettendo in discussione la capacità di azione nei riguardi degli altri; identificazione con la vittima e vissuto di impotenza connesso; spostamento dell'attenzione da sé all'altro con particolare riferimento al danno permanente subito dalla vittima di abuso, specie se questo è avvenuto in età infantile; ascolto introspettivo e ricaduta emotiva soggettiva che la visione del film può avere sollecitato.

\section{Esiti della valutazione in itinere del progetto.}

Il progetto "www...parliamone pure", come già esplicitato, è stato portato avanti in cinque diverse fasi, corrispondenti temporalmente ai seguenti periodi, legati ad esigenze della struttura penitenziaria:

- Primo periodo: maggio - ottobre 2009;

- Secondo periodo: ottobre 2009 - gennaio 2010;

- Terzo periodo: ottobre - dicembre 2010;

- Quarto periodo: marzo - giugno 2011;

- Quinto periodo: ottobre - dicembre 2012.

Per ognuna delle fasi sopra indicate, l'équipe degli operatori che ha condotto i lavori ha individuato criticità ed ha effettuato valutazioni tecnico-professionali circa risultati conseguiti ed obiettivi per le successive fasi.

Per una valutazione in itinere delle attività condotte è importante fare riferimento ad ogni succitato periodo.

\subsection{Primo periodo: maggio-ottobre 2009.}

Gli elementi di criticità individuati sono: 
1. lo svolgimento dell'attività in un contesto di istituzione totale quale quella carceraria. Si è infatti assistito ad un iniziale atteggiamento di difesa da parte di alcuni operatori, soprattutto quelli della Polizia Penitenziaria, probabilmente legato ad un loro mancato coinvolgimento nella promozione del progetto; va tuttavia rilevato che gli stessi nel corso dello svolgimento hanno progressivamente modificato l'atteggiamento originario mostrando disponibilità organizzativa in funzione della sensibilizzazione attuata in itinere;

2. difficoltà legate all'orario riservato alla conduzione dei gruppi, talora coincidente con il pranzo e con la celebrazione della S. Messa ed alle interruzioni del lavoro di gruppo da parte della Polizia Penitenziaria per comunicazioni inerenti i singoli detenuti;

3. la presenza altalenante di alcuni detenuti per diverse ragioni (scarcerazioni, trasferimenti per motivi sanitari o di giustizia, lavoro di distribuzione del vitto);

4. l'impossibilità di attuazione di alcune attività previste $\mathrm{ab}$ inizio dal progetto (attività culturali, arte terapia, azione sinergica con la Polizia Penitenziaria, supervisione, mediazione familiare).

Relativamente alle criticità suddette i professionisti impegnati nella conduzione del gruppo si sono attivati nell'affrontare dinamicamente tali difficoltà riuscendo ad individuare delle soluzioni, mentre per le attività previste ma non realizzate, ad oggi si rimane in attesa di riscontri positivi da parte della Provincia Regionale di Trapani (ente locale) a cui è stata avanzata una richiesta di finanziamento relativamente alla quale è stata verbalmente espressa disponibilità nel caso fosse possibile reperire canali adeguati di finanziamento.

Per quel che riguarda la validità del percorso finora attuato, si rilevano aspetti relativi alla partecipazione attiva di ogni singolo detenuto, alla disponibilità espressa nell'approfondire contenuti emozionali ed esperienziali via via emersi, all'apertura verso la trattazione del tema "reato".

\subsection{Secondo periodo ottobre 2009- gennaio} 2010.

In tale periodo hanno aderito i detenuti coinvolti fin dall'inizio del progetto. Si è registrata a tal riguardo, quale criticità legata all'istituto penitenziario, una variazione legata ad una rinuncia da parte di un detenuto e all'ingresso da parte di un altro detenuto della sezione protetti; si è continuato a registrare una presenza altalenante di alcuni condannati per ragioni diverse: turnazioni per opportunità lavorative interne, trasferimenti per motivi sanitari e di giustizia.

A conclusione del percorso attuato i detenuti hanno evidenziato l'importanza della esperienza di gruppo sulla loro vita detentiva. In maniera particolare hanno espresso come il lavorare in gruppo abbia restituito loro una dimensione di dignità e di rispetto come persone al di là della condanna in espiazione.

L'azione di gruppo ha agito positivamente nel contesto di una istituzione totale, quale quella penitenziaria, che nella sezione protetti offre scarse opportunità trattamentali, condizione questa percepita dai detenuti con sentimenti di abbandono e di pregiudizio. E' stato utile, in questo senso, l'ascolto e il confronto in gruppo, 
vissuto da parte dei detenuti come strumento significativo per sostenere il peso della detenzione ed al contempo elaborare considerazioni adeguate per affrontare la vita esterna.

Gli elementi di criticità hanno riguardato: la non continuità del progetto in corso; l'approvazione di una nuova normativa in materia di violenza sessuale e la restrizione dei benefici di legge.

Per quel che riguarda la validità del percorso esperienziale, gli operatori evidenziano aspetti significativi da un punto di vista trattamentale sia relativamente al percorso detentivo che in vista del re-ingresso del soggetto in ambiente libero. In particolare, $\mathrm{i}$ detenuti sono stati sostenuti e supportati nella gestione della solitudine quotidiana in sezione; si è cercato di sollecitare la sfera emotiva degli stessi, avviandoli verso il riconoscimento e la consapevolezza di emozioni e stati d'animo propri e dell'altro. L'approfondimento in tale direzione è stato ritenuto essenziale da parte dell'équipe per "guidare" i detenuti verso una esperienza di percorso terapeutico presso strutture territoriali specialistiche che gli stessi potrebbero avviare al fine della pena. Gli operatori hanno sostenuto tale orientamento nell'ottica della sicurezza sociale e della prevenzione della recidiva. Naturalmente tale percorso va ancora sostenuto e potenziato centrando l'attenzione sulla vittima, sul riconoscimento e sulla gestione della propria colpevolezza, sul danno sociale.

\subsection{Terzo periodo: ottobre- dicembre 2010.}

Gli elementi di criticità individuati sono:

- l'esiguo numero di ore a disposizione dell'équipe;

- la breve durata del modulo esperienziale;
- l'incertezza rispetto alla prosecuzione del progetto;

- l'impossibilità di attuazione di attività psicocorporee;

- la mancata realizzazione di incontri con la Polizia Penitenziaria.

Tali elementi non hanno consentito una conseguente evoluzione del percorso di rielaborazione interiore e personologica da parte dei detenuti, in una fase in cui gli stessi sono apparsi, a parere dell'équipe, predisposti a raccontare e condividere la propria esperienza deviante, contestualizzata nella loro storia di vita. Pertanto, si è auspicata la prosecuzione dell'attività progettuale ritenendo che il trattamento penitenziario dei sex-offenders potrebbe rappresentare per questi l'unica opportunità di riflessione e di rivisitazione dei loro agiti devianti, stimolando, altresì, il riconoscimento della loro eventuale problematicità psicologica e del loro bisogno di aiuto. Tale esperienza, a parere dell'équipe, rappresenta l'unico aggancio tra l'istituzione carceraria e la comunità esterna.

\subsection{Quarto periodo: marzo - giugno 2011.}

In tale periodo, la prosecuzione del progetto avrebbe potuto, dopo l'esperienza vissuta, condurre ad un confronto critico sul reato sessuale, sull'autore e sulla vittima del reato e ad abbozzare prospettive future di reinserimento sociale.

Infatti, a conclusione del percorso, l'équipe, anche in presenza di detenuti in prossimità del fine pena, ha fornito indicazioni sulle opportunità esterne di aiuto e sostegno cui 
potere fare ricorso nella fase del re-ingresso in società.

Ulteriore aspetto positivo in questa fase è stato il rapporto con la Polizia Penitenziaria; si è realizzato a tal riguardo un incontro dal quale è emersa l'importanza del lavoro sinergico, in quanto insieme attori del medesimo processo di risocializzazione. Alla Polizia Penitenziaria è stato chiesto specificatamente un ruolo di collaborazione nel prestare attenzione agli atteggiamenti mostrati dai detenuti dopo ciascun incontro. Il personale di Polizia Penitenziaria si è mostrato disponibile svolgendo un'importante azione di agevolazione dei lavori di gruppo, avviando la risoluzione di una criticità evidenziata nei precedenti periodi.

$\mathrm{Si}$ è ritenuto auspicabile che tale esperienza potesse proseguire come attività trattamentale ed al contempo come iniziativa per costituire un coordinamento che preveda un tavolo di lavoro per mettere in rete chi a vari livelli si occupa di problemi connessi ai sex offenders e poter giungere ad individuare una modalità operativa condivisa anche a livello regionale.

\subsection{Quinto periodo: ottobre - dicembre 2012.}

La valenza trattamentale del progetto risiede nel fatto che, a partire da condizioni psicologiche e socio-culturali diverse tra i membri dei gruppi, il confronto programmato e guidato, nonché il contenimento e le restituzioni hanno raggiunto l'obiettivo di costruire una matrice del gruppo in un clima di fiducia e solidarietà. Ciò ha consentito ai singoli di osservarsi, di ascoltarsi, di confrontarsi, di ripensare ai propri vissuti e alla storia deviante e di individuare le strategie non adattive agite nel loro percorso di vita. Parimenti si è avviato un percorso di consapevolezza rispetto ad una parte di sé patologica; ciò a parere dell'équipe è da considerare un utile punto di partenza per il prosieguo delle attività di realizzazione del progetto, rispondente a quanto emerso dall'analisi dei bisogni dei detenuti stessi, come rilevati in itinere nelle diverse fasi del suo svolgimento e verificato soprattutto nell'ultimo stadio delle azioni.

Resta qualche elemento di criticità:

- la discontinuità temporale con lunghe latenze tra una realizzazione e l'altra di anno in anno;

- la mancanza di condizioni di fattibilità di alcune attività previste nel progetto, quali attività culturali, arte terapia, la supervisione;

- l'esiguo numero di ore a disposizione dell'équipe che si è rilevato particolarmente negativo sia per $i$ detenuti sia per il programmato lavoro di concerto con gli operatori della Polizia Penitenziaria;

- l'incertezza rispetto alla prosecuzione del progetto.

Alla luce delle valutazioni finali e con riferimento all'evoluzione del percorso, nonostante le lunghe interruzioni tra le diverse annualità, l'esperienza si ritiene valida. La prosecuzione appare indispensabile anche per garantire il dovuto trattamento intramurario ed extramurario. In tal senso si auspica che le attività progettuali di tipo psico-sociale in atto rivolte solo al gruppo e limitate al contesto restrittivo possano riflettersi in spazi e tempi diversi con logica di continuità, ove il singolo detenuto possa essere supportato all'interno del carcere grazie al potenziamento del trattamento psicologico individuale e all'esterno con la segnalazione e presa in carico 
degli U.E.P.E. competenti nelle prime fasi del reingresso in ambiente libero con interventi di assistenza post-penitenziaria e di invio alle strutture sanitarie di riferimento.

\section{Bibliografia.}

- Chinnici G. (a cura di), Sulle tracce della pedofilia. Aspetti psicologici, criminologici, etici e giuridici, Ed. Sviluppo Solidale, Palermo, 2004.

- Gabbard G.O., Psicbiatria psicodinamica, Raffaello Cortina Editore, Milano, 1995.

- Giuffrida M.P., I Centri di Servizio Sociale dell'Amministrazione Penitenziaria, Edizioni Laurus Robuffo, Roma, 1999.

- Gruppo Piano, Relarione del Distretto sociosanitario D54 di Castelvetrano per l'Assessorato Regionale della Famiglia, delle Politiche Sociali e delle Autonomie Locali, 2001.

- Kohut H., Narcisismo e Analisi del sé, Ed. Bollati Boringhieri, Torino, 1976.

- Marmocchi P., Dall'Aglio C., Tannini M., Educare le life skills, Edizione Centro Studi Erickson, Trento, 2004.

- Martucci L., "Il trattamento in carcere degli autori di reato sessuale e i progetti strutturati di trattamento", in Pajardi D. (a cura di), Oltre a sorvegliare e punire, Giuffrè Editore, Milano, 2008, pp. 209-232.

- Ministero della Giustizia, P.R.A.P., Carta dei Servizi degli U.E.P.E. della Sicilia (ex C.S.S.A.), Next, Palermo, 2004.

- Pugiotto A.,"La Costituzione dietro le sbarre, la pena: prospettiva statica e prospettica dinamica", relazione presentata al Master II livello, Diritto Penitenziario e Costituzione, a.a. 2014/2015, Università Roma Tre, Roma, 30/01/2015.

- Ruocco M., "La visione fenomenologica della negazione nella violenza sessuale", $D a$ Persona a Persona, Rivista di Studi Rogersiani, Aprile 2014, pp. 131-143.

- Zagrebelsky G., "Che cosa si può fare per abolire il carcere", La Repubblica, 23/01/2015.

\section{Siti web consultati.}

- $\quad$ www.demo.istat.it

- www.ComunediCastelvetrano.it 